\title{
SIMULATING SOIL WATER AND SOLUTE TRANSPORT IN A SOIL-WHEAT SYSTEM USING AN IMPROVED GENETIC ALGORITHM
}

\author{
Changshou Luo ${ }^{1, *}$, Sufen Sun ${ }^{1}$, Junfeng Zhang ${ }^{1}$, Qiang Zuo ${ }^{2}$, Baoguo Li ${ }^{2}$ \\ ${ }^{I}$ Institute of Information on Science and Technology of Agriculture, Beijing Academy of \\ Agriculture and forestry Sciences, Beijing, 100097, China \\ ${ }^{2}$ College of Resources and Environment, China Agricultural University, Beijing, 100094, China \\ * Corresponding author, Address: Institute of Information on Science and Technology of \\ Agriculture, Beijing Academy of Agriculture and forestry Sciences, Beijing, 100097, China \\ Email: luocsyoupan@163.com
}

Abstract: An improved genetic algorithm was applied and examined to optimize the weights of a neural network model for estimating root length density (RLD) distributions of winter wheat under salinity stress. Thereafter, soil water and solute transport with root-water-uptake in a soil-wheat system were simulated numerically, in which the estimated RLD distributions were incorporated. The results showed that the estimated RLD distributions of winter wheat using the neural network model combined with the improved genetic algorithm, as well as the simulated soil water content and salinity distributions, were comparably well with the experimental data. The method can serve in modeling flow and transport under salinity or saline water irrigated areas.

Keywords: genetic algorithm; root length density distribution; soil water content; salinity; simulation

\section{INTRODUCTION}

Because of water resources shortage in China, study on soil water and salinity distributions on the conditions of light-saline irrigation and rootwater-uptake was flourish (Khosla, 1997). A lot of root-water-uptake models included root distribution function. Constructing empirical model or fitting measured data were the common methods used for getting distribution 
parameters (Wu, 1999). Because the model of root distribution in soil profile was not standard and it would be different according to different crop and soil environment, there was more difference between root parameters and real data. Plant roots went hand in hand with canopy (Thornley, 1998). An improved genetic algorithm was applied in this thesis. This method can numerically estimate root distribution and get relative exact parameters of root distribution surrounding salinity in indirect and easy way. Then the parameters combined with water and solute transport model to study water and solute transport. These would be significant for study on crop growth, secondary salinization prevention and light-saline utilization surrounding salinity.

\section{EXPERIMENTAL DESIGN OF WATER AND SOLUTE TRANSPORT UNDER CONDITION OF ROOT-WATER-UPTAKE}

\subsection{Experimental design}

This thesis designed winter wheat indoor soil column experiment. Cultivar was NongDa 189 (ND189). Soil was sandy soil. Bulk density was $1.64 \mathrm{~g} / \mathrm{cm} 3$. Field capacity was $0.07 \mathrm{~cm} 3 / \mathrm{cm} 3$. Soil column was polyethylene tube of $10 \mathrm{~cm}$ inner diameters. Sandy soil was loaded in hierarchy which was $5 \mathrm{~cm}$ per layer. The total height was $40 \mathrm{~cm}$. There were respectively tensiometers and salinity sensors in $5 \mathrm{~cm}, 10 \mathrm{~cm}, 15 \mathrm{~cm}, 25 \mathrm{~cm}, 35 \mathrm{~cm}$. Experimentation which was designed one controlled disposal (no salinity stress), two salinity disposal (salinity disposal 1 and salinity disposal 2) and three repeats was practiced in wheat seedling. There was no $\mathrm{NaCl}$ in nutrient solution of controlled disposal. $3 \mathrm{~g} / \mathrm{L} \mathrm{NaCl}$ was in nutrient solution of salinity disposal. $1.6 \mathrm{~g} / \mathrm{L} \mathrm{NaCl}$ was in nutrient solution of salinity disposal 2 . There were four wheat cultivars per soil column (equal to $4500000 / \mathrm{hm} 2$ in field). $3 \mathrm{~cm}$ quartz sand was used to cover sandy soil in order to prevent evaporation after seedling. Water content must be well proportioned and nutrient must be abundant. Gravimetric method was used to control water content. In the process of experiment, soil columns were taken apart once in $6 \mathrm{~d}$ in order to get concerned data of root and canopy. 


\subsection{Water and solute transport model under condition of root-water-uptake}

On the condition of vertical one-dimensional unsaturated flow, water transport fixed solution problem which included root-water-uptake in this experiment was:

$$
\begin{aligned}
& C(h) \frac{\partial h}{\partial t}=\frac{\partial}{\partial z}\left(K(h) \frac{\partial h}{\partial z}\right)-\frac{\partial K(h)}{\partial z}-S(z, t) \\
& \mathrm{h}(\mathrm{z}, 0)=\mathrm{h} 0(\mathrm{z}), \quad 0 \leq \mathrm{z} \leq \mathrm{lz} \\
& {\left[-K(h) \frac{\partial h}{\partial z}+K(h)\right]_{z=0}=E(t), t>0} \\
& \mathrm{~h}(\mathrm{lz}, \mathrm{t})=\mathrm{h} 1(\mathrm{t}), \quad \mathrm{t}>0
\end{aligned}
$$

h- soil water matrix potential, cm. z- spatial coordinate, $\mathrm{cm}$, adown. C (h)water capability, $\mathrm{cm}-1 . \mathrm{K}(\mathrm{h})$ - unsaturated hydraulic conductivity, $\mathrm{cm} \cdot \mathrm{d}-1 . \mathrm{t}-$ time ordinate, d. h0(z),h1(t)- given function (or discrete points). lz- vertical total depth in simulation zone, $\mathrm{cm} . \mathrm{S}(\mathrm{z}, \mathrm{t})$ - root-water-uptake rate, d-1. E(t)evaporation or irrigation intensity (evaporation:-, irrigation: + ), $\mathrm{cm} \cdot \mathrm{d}-1$.

On condition that taking no account of soil static water influence and sorption and ignored influence of soil temperature gradient in this experiment, vertical one-dimensional unsaturated soil water and solute transport equation was:

$$
\begin{aligned}
& \frac{\partial(\theta C)}{\partial t}=\frac{\partial}{\partial z}\left(\theta D_{s h} \frac{\partial C}{\partial z}\right)-\frac{\partial(q C)}{\partial z} \\
& C(z, 0)=C_{0}(z) \quad 0 \leq \mathrm{z} \leq \mathrm{lz}, \mathrm{t}=0 \\
& {\left[-\theta D_{s h} \frac{\partial C}{\partial z}+q C\right]_{z=0}=q_{0} C_{0}^{\prime}(t), z=0, t>0} \\
& C\left(L_{z}, t\right)=C(t) \quad \mathrm{z}=\mathrm{lz}, \quad \mathrm{t}>0
\end{aligned}
$$

C- soil salinity concentration, g.L-1. Dsh- hydrodynamic dispersion coefficient, $\mathrm{cm} 2 \cdot \mathrm{d}-1$. q- unsaturated $\mathrm{DaXi}$ flow velocity, $\mathrm{cm} \cdot \mathrm{d}-1 . \mathrm{CO}(\mathrm{z})-$ given profile concentration, $\mathrm{g} \cdot \mathrm{L}-1 . \mathrm{C}(\mathrm{t})$ - given concentration, $\mathrm{g} \cdot \mathrm{L}-1 . \mathrm{C}_{0}^{\prime}(\mathrm{t})$ irrigating water concentration, $\mathrm{g} \cdot \mathrm{L}-1$, the value was 0 on the period of evaporation. 


\section{ROOT DISTRIBUTION ESTIMATION MODEL BASED ON IMPROVED GENETIC ALGORITHM}

On the condition of crop in existence, in the simulating process of water and salute transport model, root-water-uptake source terms was the key for the accuracy of simulating soil water and salute distribution. But the model of root in soil profile was not standard and it would be different according to crop and soil environment. Based on close relationship of root and canopy, this research availed oneself of artificial neural network advantages for modeling information complex question to construct artificial neural network model in order to estimate root distribution parameters. It also took advantage of briefness, robustness and global optimization of genetic algorithm to optimize weights of artificial neural network model with improved genetic algorithm. The artificial neural network model based on improved genetic algorithm could provide root distribution parameters. Moreover, the parameters could combine with root-water-uptake model and water and solute transport model to simulate water and solute transport distribution on the condition of root-water-uptake.

\subsection{Construction of artificial neural network model for estimating root distribution parameters}

Aboveground dry matter weight and leaf area have close relation with root growth. The two parameters should be used as input variables. Because crop growth time can show crop genetic characters on the whole and soil water and salinity were the main reasons of root growth and distribution difference, crop growth time, soil matrix potential in different depth and salinity should be input variables too. Root length density in different depth was the output variable. This search used feed forward artificial neural network which included three layers (Yuan, 1999). The three layers were input-layer, out-layer and middle-layer (hidden-layer). There were connections between upper layers nerve cells and under layer nerve cells. There were no connections between same layers.

\subsection{The process of genetic algorithm optimizing artificial neural network model weights}

Genetic algorithm was a computing model which simulating natural Bioevolution. People were taking more and more attention to genetic algorithm because it was simple, robust, global search and not be limited by search 
space. Genetic algorithm has simulated propagation, mating and mutation in the process of natural selection and natural inheritance. It expressed questions as chromosomes which would form original chromosomes colonies. Then original chromosomes colonies were parked in question environment. The chromosomes which can adapt environment were selected according to the survival of the fittest and would be operated by copy, across and aberrance. Accordingly the new chromosomes colonies were brought. Circulation would be incessant until the fittest individual was selected (Chen, 1996). Finally the optimal solution was obtained. The main process of genetic algorithm to optimize artificial neural network weights in this thesis was:

1 Real coding strategies was applied in chromosomes gene coding of artificial neural network model weights.

2 Produce the original chromosomes colonies.

3 Calculate the individual fitness value of colonies.

4 Genetic operation which included turntable bet selection as well as across and mutating operation according to certain rules was conducted.

5 Recalculate the fitness value of chromosomes colonies.

6 If the search criteria were met, iteration would stop and gave the optimal solution. Otherwise, turn to step 4.

In order to prevent optimal individual to be destroyed by genetic operation, the optimal individual reservation strategy was implemented in genetic operation.

\subsection{Determinate fitness function in model}

Fitness function was a criterion to judge individual of chromosomes colonies. Genetic algorithm used fitness value to instruct search direction. The fitness function was used by us in this search as follows:

Input and output variable formed training samples to train neural network which presented by individuals of chromosomes colonies in order to calculate learning error $\mathrm{E}$ of each individual. Formula was:

$$
E=\sum_{i=1}^{n} E_{i}, \quad E_{i}=\sum_{l=1}^{m}\left(y_{l}^{i}-C_{l}^{i}\right)^{2} / 2
$$

n- training samples number. m- output units number. yil-Cil - diffirence between real output and expectation output of No. 1 when No. i sample was training. Fitness function was decided by following formula.

fs $=1 / \mathrm{E}$,

Fitness function assured error less the fitness value more large. 


\subsection{Improve genetic algorithm}

When genetic algorithm was training artificial neural network weights, crossover operator and mutation operator played the great role in optimization process. Chromosomes may be likely to converge or diverge in search space while simple genetic algorithm was optimizing artificial network weights. This caused speediness and global convergence of search under expectation. After improved crossover operator and mutation (Luo, 2000), the time of optimizing neural network weights would decrease and convergence and stability would improve. Realize concretely as follows:

Crossover operator:

If $\mathrm{x} 1, \mathrm{x} 2$ were parent individuals which were uniformly distributing random numbers in interval $\mathrm{V}=[\mathrm{xmin}, \mathrm{xmax}]$. Progeny individuals $\mathrm{z} 1, \mathrm{z} 2$ after crossover would be produced via formula as follows (Huang, 1999):

$$
\begin{aligned}
& y_{1}=\alpha x_{1}+(1-\alpha) x_{2}, \quad y_{2}=\alpha x_{2}+(1-\alpha) x_{1} \\
& z_{1}=\operatorname{MOD}\left(y_{1}, V\right), \quad z_{2}=\operatorname{MOD}\left(y_{2}, V\right)
\end{aligned}
$$

a- integer. MOD- modular arithmetic operator.

Mutation operator conformed to rules as follows (Zheng, 2000):

$$
P_{m}=0.001+N G \cdot \operatorname{cof}
$$

Pm- variance rate of present algebra. NG- continuously un-evolutional algebra since the last evolution. Cof- parametes which decided threshold of chromosomes coercive mutation (100\% mutation).

$$
\operatorname{var}=\text { rand } \cdot w \cdot \text { dyna }
$$

var- mutation variables. Rand- random number in $[0,1]$. w- a fixed value in value range of weight. Dyna- dynamic parameters which decided mutation variables var, and initializing dyna $=1.0$. if counter $>$ nochange, dyna $=$ dyna $\times 0.1$ and counter $=0$, counter was counter which used to cumulate continuously un-evolutional algebra since the last evolution. nochangeconstant used to judge alteration of dyna value.

After combination application of crossover operator and mutation operator, on the one hand distribution of progeny individuals was even in search space, on the other hand a self-adaptive mutation mechanism could be constructed. Therefore these could keep upper efficiency in the process of genetic algorithm optimizing neural network model weights all along. Evaluation could refer to reference (Luo, 2005). In conclusion, improved genetic algorithm can improve convergence in the process of genetic algorithm optimizing neural network model and reduce the time of solution. These could gain one's ends to improve efficiency of optimizing root distribution estimation model. 


\subsection{Obtain parameters of model}

In the above-mentioned experimental design, the model parameters can be obtained via following methods:

Canopy parameters measure:

Measuring frequency was once in 6d. Measuring items included dry matter and leaf area. Leaf area measure used Snapscan1236 scanner made by company AGFA of Germany. Then leaf area calculation used WinRhizo pro root analysis software made in Regent Instrument company.

Root length density measure:

Soil columns were taken apart once in $6 \mathrm{~d}$. At first cut open soil columns in lengthways. Then sandy soil with root was intercept per $4 \mathrm{~cm}$ in transverse and put in sifter. The last, water used to wash root cleanly. Snapscan1236 scanner was used to scan root and WinRhizo pro root analysis software calculate root length.

Soil salinity measure:

TYC- $\square$ soil salinity sensor was used to obtain soil salinity very day on time.

Soil matrix potential measure:

Tensiometer was used to measure soil matrix potential very day on time.

Evaporation measure:

Gravimetric method was used to measure evaporation once in $3 \mathrm{~d}$.

\section{APPLICATION AND ANALYSIS OF MODEL}

\subsection{Analysis of root distribution prediction result}

According to description of constructing artificial neural network model for root distribution parameters estimation and experiment design in this research, winter wheat root distribution estimation model under salt stress was a 13-9-10 network. Input layer was composed of growth time, aboveground dry matter, leaf area, matrix potential in 5, 10, 15, 25, 35 and salinity. Nerve cells number was 13. Output layers were composed of root length density of each $4 \mathrm{~cm}$ soil column in transverse. Nerve cells number was 10 . There were no specific prescribe to design middle layers nerve cells number in theory. The number generally decided by experience. If middle layers nerve cells were less, the model global optimization would weaken. If middle layers nerve cells were more, the model generalization would weaken. This experiment design middle layers were 9. Improved genetic algorithm was applied to optimize neural network weights in this 
experiment. Moreover, reservation strategy was applied in the experiment process. Colony scale was 31. Training precision was designed under learning error $\mathrm{E}<0.05$. After learning, the samples which had neither part nor lot in learning were estimated as follows:
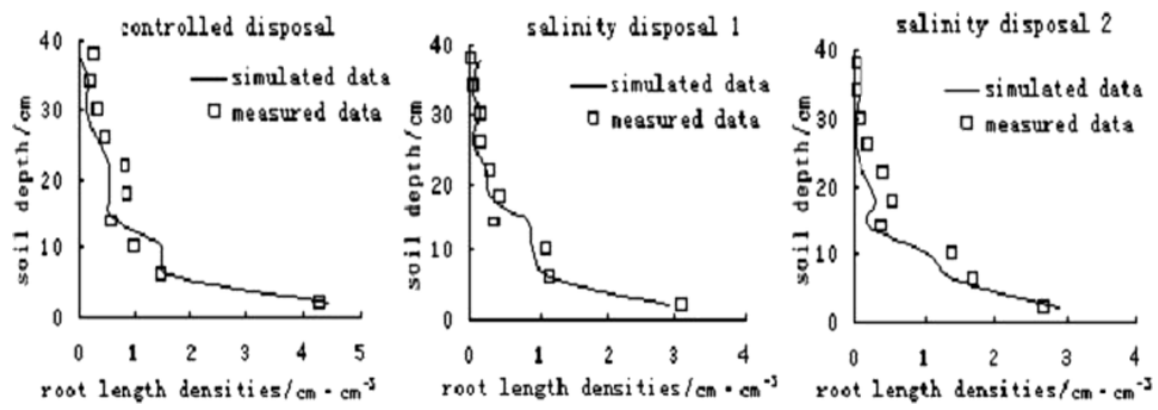

Fig. 1. Comparision between measured and estimated root length densities

Table 1. Error analysis of results between measured and estimated root length densities

\begin{tabular}{lcc}
\hline Disposal & $\begin{array}{l}\text { Average } \\
\text { relative error }\end{array}$ & $\begin{array}{l}\text { Correlation } \\
\text { factor ( } \mathrm{n}=18)\end{array}$ \\
\hline Controlled disposal & 0.24 & \\
Salinity disposal 1 & 0.26 & 0.97 \\
Salinity disposal 2 & 0.27 & \\
\hline
\end{tabular}

Fig. 1 was the comparision between measured and estimated root length densities. Fig. 1 showed holistic estimation was preferable except exceptional individuals. Moreover, while the learning samples were composed of diverse learning samples in the estimation process for diverse samples, the model estimation could be preferable generally and show good convergence and stability. Pictures indicated that root mainly distributed in $25 \mathrm{~cm}$ under ground. So we analyzed average relative error between root length densities estimated and measured data and calculated correlation factor of theirs. The result refers to table 1 . In the process of calculating average relative error, less effect of exceptional points brought on the augment of estimation data average relative error. However, the general correlation factor which was 0.97 showed model estimation generally worked well. As well as Fig. 1 showed that estimation of controlled disposal was better than salinity disposal 1 and salinity disposal 1 estimation was better than salinity disposal 2. Taking complexity and diversification of interaction between crops and environment into account, estimation result of this model was receivable. In conclusion, based on soil water parameters, salinity parameters and canopy parameters which went hand in hand with root distribution and easily be 
obtained, weights of improved genetic algorithm optimizing neural network model used to quantitatively forecast root distribution was feasible. This method can obtain parameters and root distribution data which was difficult easily.

\subsection{Construct water uptake model under salinity stress}

On the condition that salinity stress (no water stress), root-water-uptake model which was brought forward by Feddes etc was used broadly to show root-water-uptake rules. The model was:

$$
S=\alpha\left(h_{0}\right) S_{\max }
$$

S- root water uptake rate which denoted root water uptake amount in unit time and unit soil, $\mathrm{cm} 3 \cdot \mathrm{cm}-3 \cdot \mathrm{d}-1$. h0- soil water osmotic potential, $\mathrm{cm}$. Smax- maximal root water uptake rate, d-1. Smax can be obtained by following formula (Wu, 1999):

$$
S_{\max }=\frac{T_{P}(t) L_{d}(z, t)}{\int_{0}^{L_{r}(t)} L_{d}(z, t) d z}
$$

z- soil depth, $\mathrm{cm}$. Tp(t)- potential transpiration rate, $\mathrm{cm} \cdot \mathrm{d}-1$. $\mathrm{Ld}(\mathrm{z}, \mathrm{t})-$ root length densities, cm.cm-3. Ld(t)- maximal root depth, cm. a(h0)-revising coefficient of osmotic potential on the condition that not restrict water, no dimension. On the condition of no water and no nutrient stress, the better practical format of $\mathrm{a}(\mathrm{h} 0)$ as follows (Homaee, 1999):

$$
\alpha\left(h_{0}\right)=1-\frac{\alpha}{360}\left(h_{0}^{*}-h_{0}\right)
$$

$h_{0}^{*}$ - critical value of osmotic potential, $\mathrm{cm}$. a- the reducing water uptake amount while add unit conductance rate $(\mathrm{mS} \cdot \mathrm{cm}-1), \mathrm{cm}-1, \mathrm{a}=0.073$. The root-water-uptake model under salinity stress (no water stress) can be constructed by Formula (7), (9):

$$
S=\left[1-\frac{\alpha}{360}\left(h_{0}^{*}-h_{0}\right)\right] \frac{T_{P} L_{d}(z)}{\int_{0}^{L_{r}} L_{d}(z) d z}
$$

In above root-water-uptake model, root length densities derived from root distribution estimation model based on improved genetic algorithm via aboveground dry matter, leaf area, growth time, matrix potential and salinity parameters. $h_{0}^{*}$ was the measured data in reverse and the method as follows: 
Given measured water distribution of soil profile, salinity concentration distribution, root length densities distribution $\operatorname{Ld}(\mathrm{z})$ and potential transpiration rate Tp in time $\mathrm{t} 1$, $\mathrm{t} 2$, if $h_{0}^{*}$ was given an initial value, rootwater-uptake rate since t1 can be calculated by formula (10). Root-wateruptake rate used as source terms to solve water and solute transport equation in order to figure out simulation value of soil water in time $t 2$ and get square sum of the error between simulation value and measured data of soil profile water content in time t2. Then give $h_{0}^{*}$ another value, the same method was used to calculate square sum of the error until the square sum of the error was least. The value of $h_{0}^{*}$ was the parameter which we want to solve. In this experiment, the $h_{0}^{*}$ was $-560 \mathrm{~cm}$.

\subsection{Analysis of water and salinity transport simulation result}

Weights of improved genetic algorithm optimizing neural network model used to quantitatively estimate root distribution. The estimation root distribution parameters combine with root-water-uptake model to simulate water and salinity transport distribution. On the condition of salinity stress, water transport solution equation of root-water-uptake used Crank-Nicolson difference schemes to solve problems and salinity solution equation used Bresler (1973) second-order numerical differential method to solve above definite solution problems. The solving process used to simulate soil water and salinity. In the process of simulation, space step-length was $\Delta \mathrm{z}=1 \mathrm{~cm}$. Time step-length was $\Delta \mathrm{tj}+1=1.25 \Delta \mathrm{tj}$. Subscript $(\mathrm{j} . \mathrm{j}+1)$ was the ordinal number of time step-length. The control criterion of iterative water potential was $\varepsilon=0.01$. Upper boundary soil surface evaporation was $E(t)=-0.038 \mathrm{~cm} \mathrm{~d}-1$. Because limited by measured data, surface water content was given by linear extrapolation. Lower boundary was $40 \mathrm{~cm}$. Water flux and salinity flux were 0 . The maximal root depth was $L_{r}=40 \mathrm{~cm}$. Fig. 2 was a simulation result of soil water and solute transport model in soil profile. The simulation time was $3 \mathrm{~d}$. Average potential evaporation $T_{p}$ was $0.38 \mathrm{~cm} \cdot \mathrm{d}-1$. Fig. 2 showed that this method can estimate soil water distribution better under salinity stress. Moreover, except the exceptional points, the salinity simulation was generally good. In general, used as a tentative method, we thought that improved genetic algorithm quantitatively estimated root distribution and the estimation result combined with root-water-uptake model to simulate salinity stress, water and salinity transport distribution worked well. 


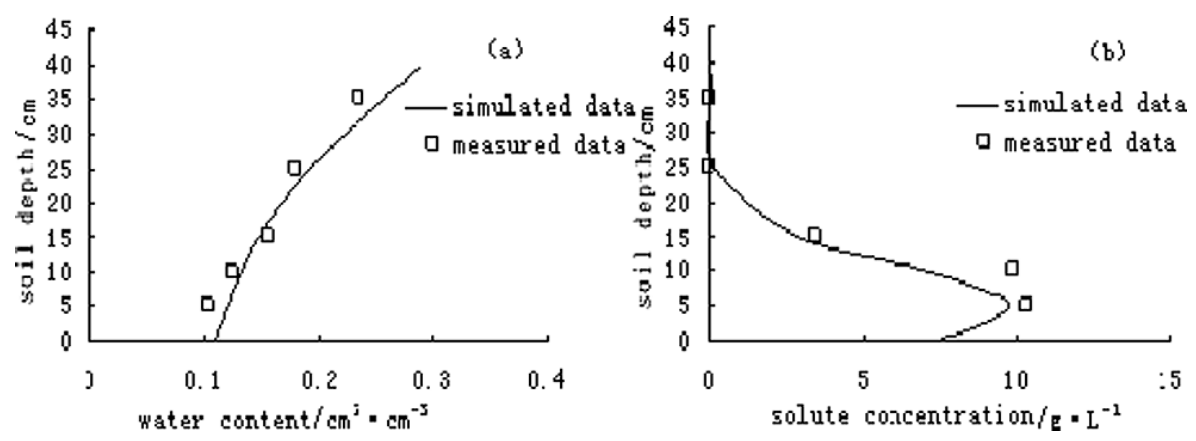

Fig. 2. Comparision between measured and estimated soil water content, salinity

\section{CONCLUSION}

Crop root-water-uptake was one of important studying problem about water movement in groundwater-soil-plant-atmosphere continuum. As well as it was the absolutely necessary data for soil water dynamic simulation in root area. A simple and practical method to get root distribution parameters was provided by improved genetic algorithm. The parameters which were obtained by this method combined with root-water-uptake model as well as water and salinity transport model can simulate soil water and solute distribution well. This method was of great significance to study relation between root distribution, salinity and crop growth as well as soil water and solute transport distribution regularity. This method can also provide decision-making gist for taking advantage of light-saline and preventing soil secondary salinization.

\section{ACKNOWLEDGEMENTS}

The accomplishment of this paper benefits from the enlightenment of professor RenLi. His numerical simulation algorithm furnishes me with inspiring source. I would also like to extend my sincere thanks to professor RenLi of Agricultural University of China.

\section{REFERENCES}

Chen GuoLiang, Wang XuFa, Genetic algorithm and its application, Post \& Telecom Press, 1996 (in Chinese)

Homaee M. Root water uptake under non-uniform transient salinity and water stress. Wageningen Agricultural University, the Netherlands, 1999, 41-54, 93-97 
Huang XiaoFeng, Pang LiDeng, Chen BiaoHua, Estimating reaction kinetics parameters with an improved real coded genetic algorithm. High Chemical Engineering transactions, 1999, 13(1):50-55 (in Chinese)

Khosla B K, Gupta RK. Response of wheat to saline irrigation and drainage, Agricultural Water Management, 1997, 32 :285-291

Luo ChangShou, Application of artificial neural network based on the genetic algorithm in predicting the root distribution. Agricultural University of China, 2000 (in Chinese)

Luo ChangShou, Zhou LiYing. Study on neural network model of improved genetic algorithm. Journal of Information, 2005, 24(5):65-66 (in Chinese)

Thornley JHM, Modelling Shoot: Root Relations: the Only Way Forword? Annal of Botany, 1998, 81:165-171

Wu J R. Zhang and S. Gui. Modeling soil water movement with water uptake by roots, Plant and Soil, 1999, 215:7-17

Yuan Zeng, Artificial neural network and its application: Tsinghua University Press, 1999, 66-70 (in Chinese)

Zheng ZhiJun, Zheng ShouQi. Adaptive genetic algorithm based on real-coded evolve neural network. Computer Engineering and Applications, 2000, (9):36-37 (in Chinese) 